health clinics in Melbourne $(n=83)$, their carers $(n=60)$ and their clinicians $(n=66)$ completed a questionnaire on the effectiveness, acceptability and side-effects of current, previous and early antipsychotic medicine. Medicine use was determined from clinical records.

Results: Patients were predominantly single middleaged women. A significant shift over time toward atypical medicine use had occurred: $66 \%$ were currently taking atypicals; compared with early medicines, current medicines were three times more likely to be atypical (odds ratio: 2.95, 95\% confidence interval: 1.48-5.88). Major discrepancies were noted in reports of medicines used between patients, carers, clinicians and clinical notes. Doctors made $61 \%$ of all recommendations for changes in previous medicines. There were few significant differences in perceived effectiveness, satisfaction and side-effects when comparing types of medicine. Health-related quality of life was associated with reported side-effects, but not with current medicine type.

Conclusions: There is a mutual lack of information and understanding about antipsychotic medicines between patients, their carers and clinicians. Greater reinforcement of provisions and incentives for collaborative treatment planning may be beneficial. Further studies of the uptake of atypical antipsychotics and their benefits should be conducted in real-world settings.

\section{A comparison of the implementation of assertive community treatment in Melbourne and London}

\section{Harvey', H Killaspy ${ }^{2,3}$, S Martino ${ }^{4}$, S White, S Johnson ${ }^{2,3}$}

Psychosocial Research Centre, Department of Psychiatry, The University of Melbourne, Melbourne, Australia; ${ }^{2}$ University College London; ${ }^{3}$ Camden and Islington Mental Health and Social Care Trust, UK; ${ }^{4}$ Private Psychiatrist, Glen Iris, Melbourne; and '5t George's University, London, United Kingdom

Background: Differences in implementation of assertive community treatment (ACT) could explain variability in reported effectiveness.

Methods: The Pan London Assertive Outreach (PLAO) studies examined ACT implementation and effectiveness amongst 24 London teams (Wright et al. 2003; Billings et al. 2003; Priebe et al. 2003). The current study gathered data on team organization, staff and client characteristics from four Melbourne ACT teams using identical participant sampling and data collection methods to the PLAO studies (except client characteristics were collected from Melbourne team staff rather than case notes).
Results: Melbourne teams were significantly different from London cluster $\mathrm{C}$ teams so comparisons were with cluster A and B teams only. All Melbourne teams worked extended hours; they took greater responsibility for dealing with crises than the London teams. Three of the four Melbourne teams achieved a majority $(>70 \%)$ of client contacts in vivo compared with only one third of the London teams. There were no significant differences between Melbourne and London teams regarding staff satisfaction and burnout. Client sociodemographic characteristics were very similar. Three quarters of all clients in both countries were admitted in the preceding 2 years but half the bed days were used in Melbourne.

Conclusions: An important difference in the implementation of ACT between Melbourne and London could be home visiting, a postulated 'active component' of models of home-based treatment. Melbourne teams may be more proactive in admitting patients at an earlier stage of relapse.

\section{The impact of the introduction of an acute sedation practice guideline}

\section{K Hirth ${ }^{1}$, A de Castella², M Dooley ${ }^{3}$, Y Hollander ${ }^{2}$}

${ }^{1}$ Alfred Hospital; ${ }^{2}$ Alfred Psychiaty; and ${ }^{3}$ Bayside Health, Monash University, Melbourne, Australia

Method: A retrospective audit of patients admitted to the Alfred Psychiatry in-patient facility from 1 October 2004 to 31 January 2006. Patient demographics, in-patient length of stay and medication usage data were extracted from the patient's medical record. High-dependency unit (HDU) length of stay was also calculated. Information regarding Code Blues, Medical Emergency Team calls, Code Greys, staff and patient incidents, and patient seclusion were also collected.

Results: There were a total of 1563 admissions to Alfred Psychiatry in-patient units during the total study period. Of these admissions, 614 included treatment in the HDU, and 313 of these HDU admissions were available for collection. Staff injuries decreased significantly from 18 in the preimplementation period to 6 in the postimplementation period $(P=0.02)$, while patient falls also decreased significantly from 17 to $1(P=0.0003)$, and patient-on-patient assaults decreased significantly from nine to zero $(P=0.007)$. The number and length of seclusions trended up but not significantly $(P=0.07)$. The use of midazolam trended down, which was clinically, but not statistically, significant. There was also a significant increase in the mean total dose of oral olanzapine used as a when-required medication from 7.2 to $22.2 \mathrm{mg}$ $(P=0.003)$. 